\title{
Development Of SSP510 Frequency Voltage Emergency Control Device
}

\author{
Qinghua $\mathrm{Si}^{1,2, \mathrm{a}}{ }^{*}$, Zhukun $\mathrm{Li}^{1,2}$, Yunsong Yan ${ }^{1,2}$, Jilin Wang ${ }^{1,2}$, Duanzhong \\ Wang ${ }^{1,3}$ \\ ${ }^{1}$ NARI Technology Development Limited Company, Nanjing 211106, China \\ ${ }^{2}$ NARI Group Corporation(State Grid Electric Power Research Institute), Nanjing 211106, China \\ ${ }^{3}$ Nanjing University of Science and Technology, Nanjing 210094, China \\ asiqinghua@sgepri.sgcc.com.cn
}

Keywords: Frequency voltage emergency control, DFT front end correction algorithm, Precise cut power load, C/S mode.

\begin{abstract}
With the construction of UHV grid and smart grid, the structure and scale of China's power grid has been greatly changed. So new requirements for frequency voltage emergency control device are presented. Architectures and features of the device is first introduced, followed by intelligent plug-management device is described, then the front end of correction algorithm based on DFT resampling is described. Additionally, the method for precise load shedding in smart substation, and the realization of standard human machine interface display and interactive method based on the C/S model are introduced. The engineering application of the device is introduced in the end.
\end{abstract}

\section{Introduction}

Frequency voltage emergency control device as the last line of defense for the safe and stable operation of power system, to ensure the safety requirements when power system is under great disturbanced, to prevent accidents, and avoid system collapse ${ }^{[1]}$. Frequency frequency voltage emergency control device which collects the frequency and voltage, and calculates the rate of change of frequency and voltage, when the system frequency or voltage drop, timely removes of a sufficient number of relatively minor user load, while the uninterruptible power supply to important users, in order to ensure system security and stable operation of the power system to prevent the expansion of the accident ${ }^{[2]}$.

With the continuous development of power system, for frequency voltage emergency control device, new requirements are proposed. The existing frequency voltage emergency control device can not fully meet the needs of the user, primarily in the following four areas: First of all, traditional frequency voltage emergency control devices can not meet the requirements of intelligent substation, poor adaptability. The second, when the grid frequency deviation from the nominal frequency is large, the error of the calculated voltage will be relatively large, causing the voltage emergency control function not to be used or the device to be mistaken ${ }^{[3-5]}$. The third, open-loop control methods, can not grasp the real-time operational status of the load line, and can not achieve precise control ${ }^{[6]}$. Finally HMI custom development, long cycle, interface unsightly, complicated operation, experience poor ${ }^{[7]}$.

With the development of smart grid, increasingly large scale system, operation mode and dynamic characteristics are more and more complex, therefore, the development of a high reliability, safety and has certain forward-looking frequency voltage emergency control device is very necessary.

\section{System Architecture}

Frequency voltage emergency control device has a large amount of data acquisition, high precision acquisition, computationally intensive, more amount of control, so SSP510 frequency voltage emergency control device is modular designed, including decision module, sampling calculating module, into module, out module, communication module, man-machine interface (HMI) module parts and so on. High speed synchronous serial communication technology based on field programmable gate array (FPGA) is adopted, and the speed can reach $32 \mathrm{Mbit} / \mathrm{s}$. Modules are 
connected through the chassis backplane bus (LVDS, RS485, CAN) or optical fiber. The structure diagram of the device is shown in Figure 1.

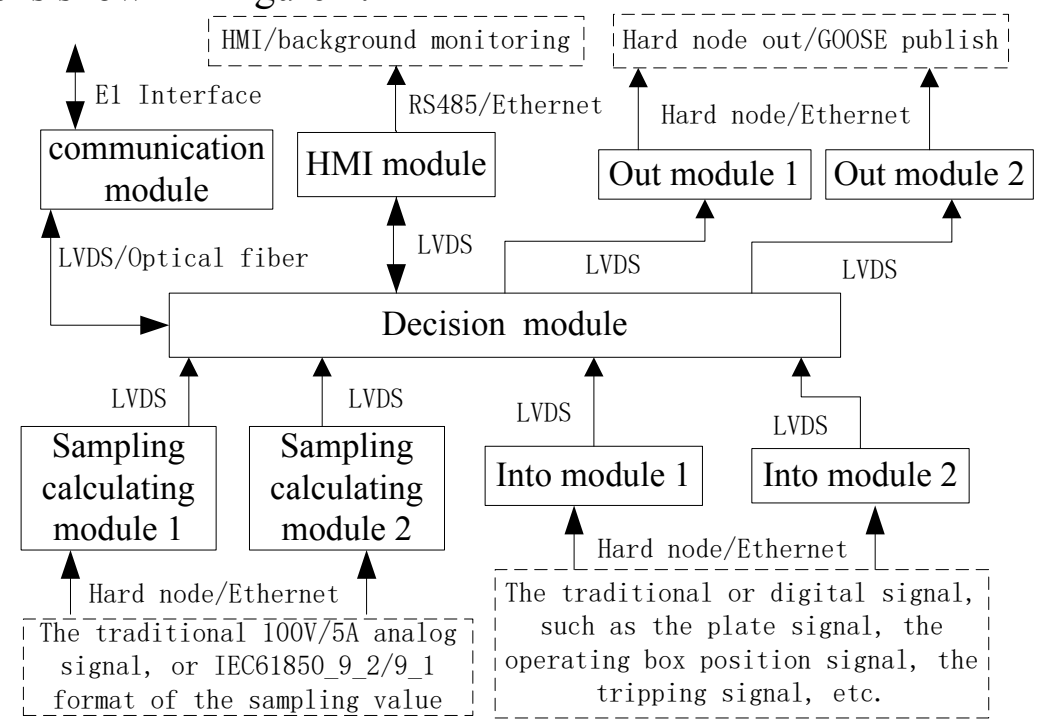

Fig. 1. system structure diagram of SSP510

The decision module is the nerve center of the device, collected information of all sampling calculation modules and into modules, and does a comprehensive analysis, to make the final judgment and the decision to out modules and communication module. The sampling calculation module is divided into the traditional analog sampling calculation module and the IEC61850 SV digital sampling and calculation module. The data acquisition, calculation and fault diagnosis of the voltage and frequency are completed by the sampling calculation module. The into module is divided into the traditional into module and the GOOSE subscribe module, for the collection of all kinds of input information, the acquisition of information including plate signal and operation box position signal, protection tripping signal etc.. The out module is divided into traditional out module and GOOSE publishing module, which receives control information sent by the decision module, through hard contact way of tripping outlet or the trip command to GOOSE network. The communication module is responsible for the communication with the remote automatic safety device, obtaining the operating condition of the remote device, achieving the distance cutting generator or cutting load. Human machine interface module provides human machine interface and communication with background management system.

With this kind of structure design, it can provide the hardware foundation for the intelligent management of the module, and can be flexible to configure different types and quantities of modules to meet the needs of different projects.

\section{Device Characteristics}

The device has a low frequency, low voltage, over-frequency and over-voltage protection function, which judges frequency and voltage accident, when the frequency and the voltage is abnormal, and quickly takes the control measures quickly to cut load line or generator. The low-frequency or low-voltage is respectively provided with 5 independent basic wheels, 3 independent special wheels and 2 accelerating wheels. When the system frequency drops quickly, the device has the function of accelerating the cutting load according to $\mathrm{df} / \mathrm{dt}$, in order to stop the decline in frequency as soon as possible. When the system voltage drops too fast, according to the $\mathrm{du} / \mathrm{dt}$ accelerate the cutting load line, as soon as possible to stop the system voltage drop, avoid voltage collapse accident, ensure the voltage stability.

The mal operation and the rejection can be effectively prevented by using many different conditions and multiple locking mechanisms. The device also has the following characteristics: intelligent management method of modules; DFT front end correction algorithm based on resampling; 
in smart substation it can achieve precise load shedding; standardized human computer interaction is achieved based on the $\mathrm{C} / \mathrm{S}$ model.

Intelligent Management Method Of Modules. For the traditional frequency and voltage emergency control device, the function and communication topology of the internal module of the chassis are basically fixed. In the smart substation, the hardware implementation faces the following challenges: in traditional substation, smart substation, semi smart substation, etc. different customized applications, how to realize fastly in specific engineering applications; in electrical compatibility, data communication, configuration management, how to manage the interface of traditional module and the digital mode, and how to realize the high speed real time interaction between the various modules when the project is complex, and so on.

An important basis for the effective solution of these problems is: more standardized chassis and intelligent modules. The modular intelligent management method is adopted, which abstracts the structure of the chassis into the physical layer, the link layer, the network layer. The physical layer provides the intelligent modules connected, EMC shielding, multi-channel power supply, power signal transmission, information and communications. The link layer provides multiple connection on BLVDS, LVDS, CAN etc. in a number of ways such as bus and star topology. The network layer uses the module internal FPGA by the data exchange area mapped to a unified memory space, to achieve a multi-node, multi-channel communications for automatic data exchange, and to make the CPU only need to read and write the specific memory in the application layer.

The method of three layers of the software and hardware design achieves standardization and intelligent in the chassis and module management. Each module is compatible with others in the chassis to improve the allocative efficiency, and enhance the system scalability.Traditional module, digital module interactive data unified management, makes algorithm design more standardized, and enhances the reliability of the device. Through the simple configuration file, the communication bandwidth can be flexibly allocated, which improves the communication efficiency. In short, the intelligent management method of the module, makes the software development more simple and reliable, and improves the adaptability of the device to different engineering requirements.

DFT Front End Correction Algorithm.Root mean square (RMS) and fast Fu Liye transform (DFT) are widely used in the secondary devices. However, they have a relatively large calculation error in the case of frequency offset ${ }^{[3-5]}$.

At present, the compensation of the error of DFT algorithm is the back end correction algorithm. There is a common problem in this algorithm. It implies a hypothesis, that in a computing period, when the frequency is calculated, the effective value is assumed constant; and when the effective value is calculated, the frequency is assumed constant. This algorithm is faced with a contradiction: to ensure the accuracy of the calculation, the number of iterations is not fixed, resulting in the calculation time is not determined; to ensure the calculation time, the accuracy can not be determined $^{[3-5]}$. If the calculation time is not determined, the reliability of the device can not be guaranteed; if the calculation accuracy is not determined, the performance of the device is reduced.

A new method of DFT front end correction algorithm is adopted, which is used to balance the accuracy and time. First, the frequency is calculated, then sampling value is modified according to the frequency, at last the effective value is calculated. The flow chart of the algorithm is shown in Figure 2.

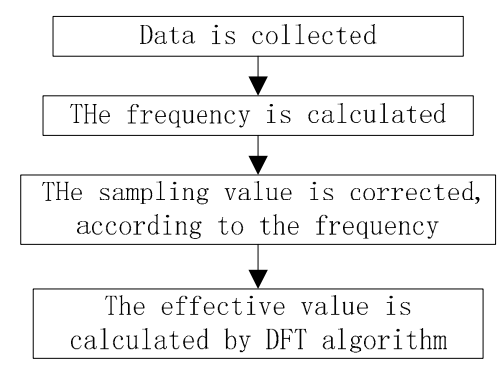

Fig. 2. Flow chart of the DFT front end correction algorithm

The comparison results of the algorithm and the RMS algorithm are shown in table 1 . The calculation time of this method is determined, and the accuracy is high, and the calculation error is 
less than $5 / 1000$ in $35 \mathrm{~Hz}-75 \mathrm{~Hz}$. Using this algorithm, the voltage emergency control function is still available when the grid frequency deviates from the rated frequency largely.

Table 1. Comparison between the DFT front end correction algorithm and the RMS algorithm

\begin{tabular}{|c|c|c|c|c|c|}
\hline \multirow{2}{*}{$\begin{array}{c}\text { Frequency reference } \\
\text { value (Hz) }\end{array}$} & $\begin{array}{c}\text { Voltage reference } \\
\text { value (Hz) }\end{array}$ & \multicolumn{2}{|c|}{$\begin{array}{c}\text { DFT front end correction } \\
\text { algorithm }\end{array}$} & \multicolumn{2}{c|}{ RMS } \\
\cline { 3 - 6 } & & Value (V) & Error(V) & Value(V) & $\begin{array}{c}\text { Error } \\
(\text { V) }\end{array}$ \\
\hline 35.000 & 100.0 & 99.8 & 0.2 & 112.3 & 12.3 \\
\hline 40.000 & 100.0 & 99.8 & 0.2 & 110.3 & 10.3 \\
\hline 45.000 & 100.0 & 99.9 & 0.1 & 106.0 & 6.0 \\
\hline 50.000 & 100.0 & 100.0 & 0 & 100.0 & 0 \\
\hline 54.000 & 100.0 & 99.9 & 0.1 & 95.9 & 4.1 \\
\hline 60.000 & 100.0 & 99.8 & 0.2 & 92.1 & 7.9 \\
\hline 70.000 & 100.0 & 99.8 & 0.2 & 88.6 & 11.4 \\
\hline 75.000 & 100.0 & 99.8 & 0.2 & 86.3 & 13.7 \\
\hline
\end{tabular}

Precise Load Shedding In Smart Substation. The open-loop control method is still widely used in frequency voltage emergency control device in cutting load loop, which does not collect the line power, making it impossible to know the changes of the load line, and therefore can not accurately cut load line. When the power fluctuates or the line state changes, it may cause the shortage or excessive of cutting load line or even lead to the expansion of the power grid fault.

In traditional substation, there may be more to dozens of load lines, if the function to achieve precise load shedding, all the lines need access to frequency voltage emergency control device. But in the actual engineering application, due to the design size, economic and other factors, this method is unable to achieve. In smart substation, due to SV network bandwidth and the limit of communication and computing processing, the method can not realized in engineering in quite a long period, which obtains load line power through the network of SV.

In smart substation, if GOOSE application range is extended, the use of GOOSE network transmission load line power, precise load shedding can be achieved on the premise of not increasing investment $^{[6]}$. The load line power is added into the corresponding GOOSE control block. By means of the GOOSE subscription method, the load power and the line status information are obtained. According to the amount of load shedding required, the lines are cut intelligently, so as to achieve accurate load shedding. The flow chart of the method is shown in Figure 3.

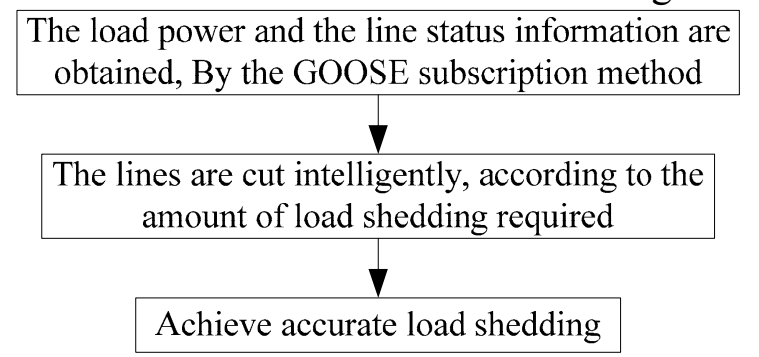

Fig. 3. Flow chart of the precise load shedding

The performance of the SSP510 frequency and voltage emergency control device has been upgraded with a very small economic cost, by means of the GOOSE subscription method. This way to meet the requirements of future device technology upgrade.

Standardized Human Computer Interaction Is Achieved Based On The C/S Model. The development of the HMI is usually very close with the platform of the frequency voltage emergency control device, and it is often the full custom development. Due to the difficulty of the development, there are many limitations in the size of the display, the richness and the friendliness of $\mathrm{HMI}^{[7]}$. With the development of smart grid and industry standards, the requirements of HMI also continues to 
increase, mainly reflected in: show more intuitive, rich, more convenient way to interact, the more powerful data management, and so on ${ }^{[7]}$.

A mothod is adopted based on $\mathrm{C} / \mathrm{S}$ mode to achieve standardized human computer interaction. The device is divided into the device main body and the HMI module, which are hardware independent, only through a cable connected; standardization of HMI data are extracted and mapped to the unity of Modbus address space; the device main body as the server provided the data access port; HMI module as the client, through the Ethernet and RS-485 link, by Modbus protocol for data access; the HMI module is independent of the realization of data analysis, display, input and output and so on. The implementation flow chart of the method is shown in Figure 4.

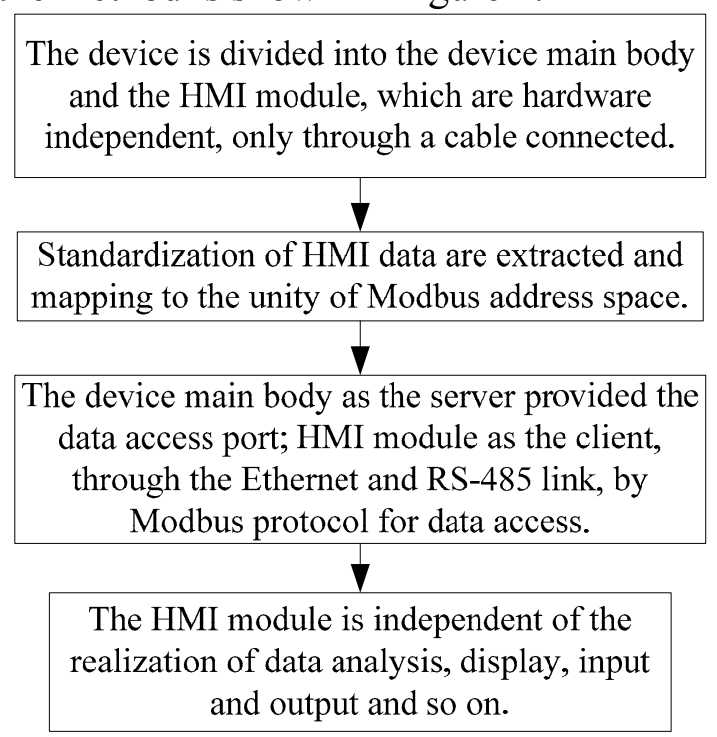

Fig. 4. Flow chart of the standardized human computer interaction

Industrial touch screen was chosen as the HMI module, display and keyboard function. The HMI module and the device main body are decouple fully, reducing the complexity of the electromagnetic compatibility of the device and structure design. The device main body handles only a small amount of data information. The complex graphics display process is transferred to the HMI module. More advanced technology of industrial touch screencan can be fully utilized, such as color display, high-resolution, large-size, touch control and so on. It can meet the reliability and practicality at the same time, effectively save the cost of developing and maintaining, shorten product development cycle, enhanced ability to upgrade.

\section{Test And Engineering Application}

In the National Relay Protection and Automation Equipment for Quality Supervision and Inspection Center, SSP510 frequency voltage emergency control device was successfully through the electromagnetic compatibility tests and the type tests. The experimental results show that the device with high precision and high reliability, can rightly judge various accidents such as low frequency, high frequency, low voltage, high voltage accident, etc., and quickly take proper control measures and ensure the safe and stable operation of the power system.

The device has been applied in many places, with fault of power system judged rightly many times. The device has never occurred mis-operation or refuse-operation, to effectively play the role of a third line of defense in the power system for reliable electricity sent out.

\section{Conclusions}

SSP510 frequency and voltage emergency control device has high precision, intelligent features to meet the requirements of modern power grid, and provides a strong guarantee for the safe and stable operation. The device has the following characteristics: the intelligent management method of the module, improves the adaptability of the device to different engineering requirements; DFT front end 
correction algorithm is adopted, improving the accuracy of effective value under the condition of frequency offset; precise load shedding is achieved by means of the GOOSE subscription line power method; standardized human computer interaction is achieved based on the $\mathrm{C} / \mathrm{S}$ model, shorten product development cycle, enhanced ability to upgrade.

In power system, if a sufficient number of SSP510 frequency voltage emergency control device is reasonable configuration, reliable third line of defense can be established, avoiding or mitigating the accident of power gird, greatly improving power generation and transmission capacity, generating huge economic benefits and social benefits.

\section{Acknowledgements}

This work was financially supported by State Grid Corporation of China (Key Technology of security and stability control device in smart grid).

\section{References}

[1] Guide on Security and Stability DL 755-2001 for Power System, China Electric Power Press, (2001), in Chinese.

[2] SUN Yujun, LING Haiyan, CHEN Xiong. Design and Implementation of the Configuration Software for SCS-200A Frequency and Voltage Emergency Control Device, J. Jiangsu Electrical Engineering, 2010, 29(6): 32-34. in Chinese.

[3] Jun-Zhe Yang and Chih-Wen Liu. A precise Calculation of Power System Frequency and Phasor. IEEE Trans On Power Delivery, Vol.15,No.2,April 2000:494-499.

[4] LI Jian, XIE Xiaorong, HAN Yingduo. Some Key Issues of Synchrophasor Measurement, J. Automation of Electric Power Systems, 2005,29(1):45-76. in Chinese.

[5] WANG Maohai, SUN Yuanzhang. A DFT-based Method for Phasor and Power Measurement in Power System, J. Automation of Electric Power Systems, 2005,29(2):20-24. in Chinese.

[6] LI Zhukun, XUAN Xiaoqing, YE Zhenfeng, LI Xuerning. Technical Ideas of Low Frequency and Low Voltage Load Shedding Devices in Smart Substation Architecture, J. Automation of Electric Power Systems, 2013，37(10): 19-22. in Chinese.

[7] Qinghua Si, Haibo $\mathrm{Xu}$, Jun $\mathrm{Xu}$, Gaoyang Xu, Zhenfeng Ye. Application of Modbus protocol in the automatic safety device, J. Advanced Materials Research, Vols. 1070-1072 in 2015 with the title Applied Energy and Power Engineering IV. 